\title{
Green Collaboration in Cognitive Radio Cellular Networks with Roaming and Spectrum Trading
}

\author{
Lokman Sboui $^{1}$, Hakim Ghazzai ${ }^{2}$, Zouheir Rezki ${ }^{1}$, and Mohamed-Slim Alouini ${ }^{1}$ \\ ${ }^{1}$ King Abdullah University of Science and Technology (KAUST), Thuwal, Makkah Province, Saudi Arabia, \\ \{lokman.sboui, zouheir.rezki, slim.alouini\}@ kaust.edu.sa \\ ${ }^{2}$ Qatar Mobility Innovations Center (QMIC), Doha, Qatar, \\ hakimg@qmic.com
}

\begin{abstract}
In this paper, we propose a new cognitive cellular network architecture based on the coexistence of primary and secondary networks, (PN) and (SN), respectively. The PN aims to minimize its energy consumption by switching off the maximum number of its BSs and offloading its users to the SN's infrastructure to maintain its QoS. In return, the PN pays a roaming price and permits the $\mathrm{SN}$ to share or lease the spectrum at a certain price. We propose a low-complexity algorithm allowing the PN to minimize its energy consumption by selecting a suboptimal combination of active base stations. Our algorithm also optimizes the resource allocation of the $\mathrm{SN}$ to maximize its total sumrate while respecting the minimal profit constraints for both networks. In the numerical results, we show that our proposed algorithm achieves close performances to the optimal exhaustive search algorithm. In addition, we investigate the impact of various system parameters in the collaboration decision.
\end{abstract}

Index Terms-Green cellular networks, underlay cognitive radio, operators collaboration.

\section{INTRODUCTION}

The evolution of wireless communications has led to multiple challenges related to the increasing demand for data rate, coverage, reliability and energy efficient communication [1]. These challenges need to be considered in the next fifth generation $(5 \mathrm{G})$ of cellular networks in order to meet its requirements [2]. One of these major challenges is to cope with this exponential growth of networks' energy consumption that currently constitutes a crucial global concern for ICT and communications fields. Indeed, mobile operators are required to reduce their energy consumption to not only achieve environmental objectives but also economic ones as about half of their expenses are coming from electricity bills. Consequently, many mobile operators tend recently to reduce their energy consumptions by adopting energy-efficient schemes [3].

Another issue is the inefficient spectrum allocation controlled by the regulatory organizations like the Federal Communications Commission (FCC) [4]. The spectrum is becoming more and more a scarce resource that prevents new wireless technologies to evolve rapidly. In traditional cellular networks, in order to satisfy the increasing data rate demand of mobile users, the operators tend to either buy more bandwidth with millions of dollars or deploy new spectral efficient technologies such as sophisticated modulation or MIMO. However, theses options come with either high cost or high complexity. To overcome this issue, the concept of cognitive radio (CR) introduced by Mitola in [5], presents a flexible spectrum access

This work was made possible by NPRP grant \# 6-001-2-001 from the Qatar National Research Fund (A member of The Qatar Foundation). The statements made herein are solely the responsibility of the authors for non-licensed/secondary users (SUs) while respecting the licensed/primary users (PUs) quality of service (QoS). In [6], [7], the CR networks were characterized by two main features: i) cognitive capability through sensing and learning from the radio environment ii) reconfigurability referring to the ability to dynamic access to the spectrum and the ability of transmitting on various frequencies. In the cellular networks framework, related paradigms such as spectrum sharing or spectrum trading can be adopted to have a convincement solution to the increasing demand for bandwidth. The concept of spectrum trading in a CRN framework is described as follows: the PU's share, lease, or sell their vacant spectrum with a certain price and the SU's share, rent, or buy the available spectrum resources. In [8], the authors study the cooperation between two operators in which the objective is to minimize the energy cost. However, both operators had equal priority.

In this paper, we focus on the cooperation between a primary network $(\mathrm{PN})$ benefiting from a high priority and a secondary network (SN) aiming to exploit the primary spectrum opportunistically. In other words, the available resources (spectrum, power, etc.) are used to first serve the PN users then the remaining resources are used to serve the SN users. In our study, we propose a collaboration scheme between a green PN and a SN accepting to serve offloaded users. The $\mathrm{PN}$ focuses on activating the minimum number of base stations (BSs) while offloading its users to the SN's infrastructure. In return, the SN accepts to serve PUs in priority while enjoying the leased spectrum to maximize its total secondary sum-rate. However, such collaboration can only be possible if the profits and QoS of both networks are not affected. Therefore, we propose a low-complexity algorithm to solve our formulated optimization problems aiming to achieve the primary and secondary objectives while taking into account the energy, roaming, and spectrum sharing costs. Finally, we show that our low-complexity algorithm achieves performance close to those of the Exhaustive Search (ES) method and outperforms the performance of a previously proposed on/off switching algorithm. Furthermore, we identify some criteria promoting the green collaboration for cognitive radio networks.

The rest of this paper is organized as follows. In Section II, the system model is described. In Section III, the problem formulation is described. The proposed PN management and the SN power allocation schemes are presented in Section IV. Numerical results are presented in Section V. Finally, the paper is concluded in Section VI. 


\section{System MODEL}

We consider a geographical area served by two cellular networks: the PN and the SN. the PN is operating on the licensed bandwidth while the SN is aiming to communicate opportunistically while sharing the primary bandwidth. We denote by $N_{\mathrm{BS}}$ the number of BSs assumed to be the same for both networks. Both the PN and the $\mathrm{SN}$ are deployed in a hexagonal cells model. Each BS adopts a tri-sectoral transmission in which the area covered by the BS is divided into three equal areas. In order to have the minimum interference, the secondary BSs are deployed such as the distance between the secondary BS and the closest primary BS is equal to the cell radius. In other words, the secondary BSs are deployed in the intersection of three primary cells. The BSs are placed to serve $\mathcal{P}$ PUs and $\mathcal{S}$ SUs distributed randomly with coordinates $x$ and $y$ according to a given joint probability density function (pdf) denoted by $f_{p}(x, y)$ and $f_{s}(x, y)$, respectively. The proportion of PUs in a sub-region denoted by $\omega$ of the considered region denoted by $\Omega$ (i.e., $\omega \subseteq \Omega$ ) is computed as $\iint_{\omega} f_{p}(x, y) d x d y$. For instance, the total number of PUs in this sub-region $\omega$ is denoted by $\mathcal{P}_{\omega}$ and is given by:

$$
P_{\omega}=\left\lceil\mathcal{P} \iint_{\omega} f_{p}(x, y) d x d y\right\rceil .
$$

where $\lceil$.$\rceil denotes the ceiling function. We denote by P_{\mathrm{x}, j}$ the consumed power of the $j^{\text {th }}$ active BS belonging to the network $\mathrm{x}$ where $\mathrm{x} \in\{\mathrm{p}, \mathrm{s}\}$ depending on the considered network (i.e., $\mathrm{p}$ for the $\mathrm{PN}$ and $\mathrm{s}$ for the $\mathrm{SN}$ ). This power is computed as follows [9]:

$$
P_{\mathrm{x}, j}=a P_{\mathrm{x}, j}^{(\mathrm{tx})}+b,
$$

where the coefficient $a$ denotes the power consumption that scales with the transmitted power due to the amplifier and the feeder losses. The parameter $b$ models the offset of site power consumed independently of the radiated power. This parameter involves, mainly, the signal processing (analog to digital converters (ADC), filters, mixers, amplifiers, etc.), battery backup, and cooling. In (2), $P_{\mathrm{x}, j}^{(\mathrm{tx})}$ denotes the transmitted power of the $j^{\text {th }}$ BS which depends on the number of users served by this BS (denoted by $\mathcal{P}_{j}$ or $\mathcal{S}_{j}$ for the PN or the $\mathrm{SN}$, respectively). This transmit power is expressed as follows [10]:

$$
P_{\mathrm{x}, j}^{(\mathrm{tx})}=\sum_{i=1}^{\mathcal{P}_{j}} \frac{P_{\min }}{K} d_{j, i}^{\nu} h_{j, i}^{\mathrm{x} \rightarrow \mathrm{y}},
$$

where $P_{\min }$ is the minimum power to be received by each user in order to ensure the required QoS, $K$ is a parameter representing the effects of BS antenna settings, carrier frequency and propagation environment, $\nu$ is the path loss exponent, $d_{j, i}$ denotes the distance between of the $j^{\text {th }}$ BS of network $\mathrm{x}$ and the $i^{\text {th }}$ user connected to this $\mathrm{BS}$, and $h_{j, i}^{\mathrm{x} \rightarrow \mathrm{y}}$ models the fastfading effect of the channel between the $j^{\text {th }}$ BS of network $\mathrm{x}$ and the $i^{\text {th }}$ of network y. The parameter $\epsilon_{j}^{(\mathrm{x})}$ is a binary variable representing the status of the $j^{\text {th }}$ BS of network x: if BS $j$ is switched on, $\epsilon_{j}^{(\mathrm{x})}=1$, otherwise, $\epsilon_{j}^{(\mathrm{x})}=0$ and $P_{\mathrm{x}, j}=0$. We assume that each BS cannot simultaneously serve a number of users exceeding $\bar{C}_{\mathrm{x}}$ such that $\mathcal{P}_{j} \leq \bar{C}_{\mathrm{p}}$ for the PN and $\mathcal{S}_{j} \leq \bar{C}_{\mathrm{s}}$ for the SN.

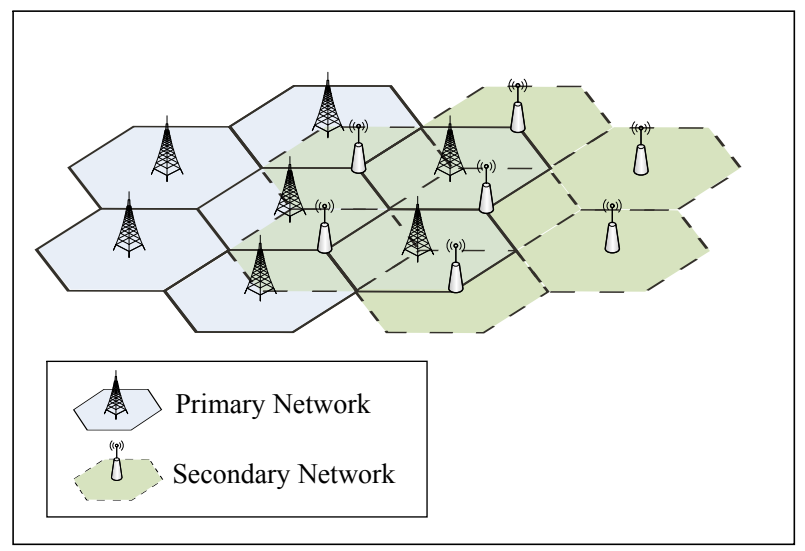

Fig. 1: Primary and secondary base stations distribution.

\section{Problem Formulation}

Our objective is to investigate the mobile operator collaboration between the PN and the SN. This collaboration is performed independently at each BS depending on the system parameters by following one of the two spectrum trading approaches:

- Spectrum sharing: This approach is based on the underlay CR scenario where the $\mathrm{SN}$ is allowed to share the primary bandwidth by transmitting over the free and non-free subcarriers while causing interference not exceeding a certain level imposed by the PN. In return, the $\mathrm{SN}$ pays a spectrum sharing price, denoted by $p_{\mathrm{ss}}$, to the PN whenever the SN causes interference to primary communications (e.g., when the corresponding PN's BS is active).

- Spectrum leasing: In this approach, the PN can exploit the existence of the secondary infrastructure in order to ensure energy savings. If the collaboration is beneficial for both networks, the PN can decide to turn off a BS and offload its users to the secondary BS while leasing the total bandwidth to the secondary transmission. the SN's task is first to ensure the PUs connectivity, then to maximize its secondary sum-rate while paying a spectrum leasing price, denoted by $p_{\mathrm{sl}}$. In return, the PN pays a roaming cost.

We assume that both spectrum sharing and leasing prices are the same for all BSs. Both networks collaborate together in order to decide either to turn off a BS or to keep it active such that their utility functions are optimized. We assume that the optimization is performed by a third-party that can be seen as a broker. This broker proposes the best solution to both the PN and the SN depending on the system parameters and indicates whether the collaboration is possible or not. If not, the PN operates using all its BSs, and the SN operates under the spectrum sharing scenario for all the BSs. In the following subsections, we describe the objectives of both the PN and the $\mathrm{SN}$ by formulating their corresponding optimization problems.

\section{A. Primary Network}

The PN's objective is to ensure energy saving by switching off the maximum number of BSs without affecting two constraints: the QoS and its profit. The QoS is satisfied when the 
users in outage rate are less than a certain outage probability threshold. A user is considered in outage when it is not being allocated to any BS (i.e., primary or secondary BS). Thus, the PN QoS constraint is written as

$$
\frac{\mathcal{P}-\sum_{j=1}^{N_{\mathrm{BS}}}\left(\mathcal{P}_{j}+\mathcal{P}_{j}^{(\text {roamed })}\right)}{\mathcal{P}} \leq P_{\text {out }} .
$$

where $P_{\text {out }}$ is the percentage of users allowed to be in outage. On the other hand, the PN's profit, denoted by $\Pi_{p}$, is expressed as

$$
\begin{aligned}
\Pi_{\mathrm{p}}=\sum_{j=1}^{N_{\mathrm{BS}}} \Pi_{\mathrm{p}, j}= & \underbrace{\left(\sum_{j=1}^{N_{\mathrm{BS}}} p_{\mathrm{op}}\left(\mathcal{P}_{j}+\mathcal{P}_{j}^{\text {(roamed })}\right)\right)}_{\text {Service revenue }} \\
& +\underbrace{\sum_{j=1}^{N_{\mathrm{BS}}}\left(1-\epsilon_{j}^{(\mathrm{p})}\right)\left(p_{\mathrm{sl}}-p_{\text {roam }} \mathcal{P}_{j}^{(\text {roamed })}\right)}_{\text {Collaboration profit }} \\
& +\underbrace{\sum_{j=1}^{N_{\mathrm{BS}}} \epsilon_{j}^{(\mathrm{p})}\left(p_{\mathrm{ss}}-p_{\mathrm{e}} P_{\mathrm{p}, j}\right)}_{\text {Non-collaboration profit }},
\end{aligned}
$$

where $\Pi_{\mathrm{p}, j}$ is the profit gained by the PN using the $j^{\text {th }} \mathrm{BS}$, where $p_{\mathrm{op}}$ is the operation price paid by all served users of the PN independently of the serving infrastructure, $\mathcal{P}_{j}^{\text {(roamed) }}$ is the number of roamed users offloaded from the $j^{\text {th }}$ BS to the neighbor $\mathrm{BS}$ belonging to the $\mathrm{SN}, p_{\text {roam }}$ corresponds to the price paid by the $\mathrm{PN}$ for the $\mathrm{SN}$ roaming service, and $p_{\mathrm{e}}$ represents the energy cost that scales with the BS power consumption during a unit of time. Note that if $\epsilon_{j}^{(\mathrm{p})}=1$, the operator pays the energy cost and gains the spectrum sharing revenue since the $j^{\text {th }} \mathrm{BS}$ is active. However, if the $j^{\text {th }} \mathrm{BS}$ is turned off, i.e., $\epsilon_{j}^{(\mathrm{p})}=0$, the operator pays the roaming service and gains the spectrum leasing revenue. The objective of the $\mathrm{PN}$ is to determine which BS should be turned off in order to reduce its energy consumption, denoted by $\mathcal{E}_{p}$, while ensuring a certain minimum profit denoted by $\Pi_{\mathrm{p}}^{\mathrm{min}}$. Hence, the PN's optimization problem can be formulated as follows

$$
\underset{\mathcal{P}_{j}^{\text {(roamed) }}, \epsilon_{p} \in[0,1]}{\operatorname{minimize}} \mathcal{E}_{p}=\sum_{j=1}^{N_{\mathrm{BS}}} \epsilon_{j}^{(\mathrm{p})} P_{\mathrm{p}, j} .
$$

Subject to:

$$
\begin{gathered}
\text { - } \frac{\mathcal{P}-\sum_{j=1}^{N_{\mathrm{BS}}}\left(\mathcal{P}_{j}+\mathcal{P}_{j}^{(\text {roamed })}\right)}{\mathcal{P}} \leq P_{\text {out }}, \\
\text { - } \quad \Pi_{\mathrm{p}} \geq \Pi_{\mathrm{p}}^{\min },
\end{gathered}
$$

where (7) and (8) represent the QoS and profit constraints, respectively.

\section{B. Secondary Network}

As per the cognitive concept, the main objective of the $\mathrm{SN}$ is to maximize its users' sum-rate, denoted by $\mathcal{R}_{s}$ by either sharing the primary channel in an underlay mode or freely exploiting the channel after serving PUs in a spectrum leasing scenario. Hence, the SN optimization problem is given as follows

$$
\begin{aligned}
& \underset{\boldsymbol{\pi}, \boldsymbol{P}}{\operatorname{maximize}} \mathcal{R}_{s}=\sum_{j=1}^{N_{\mathrm{BS}}}\left(\sum_{v \in \mathcal{I}_{j}} \log _{2}\left(1+\pi_{s, j}(v, i) P_{\mathrm{s}, j}(v) h_{j, i}^{s \rightarrow s}(v)\right)\right. \\
& \left.+\sum_{v \in \overline{\mathcal{I}}_{j}} \log _{2}\left(1+\frac{\pi_{s, j}(v, i) P_{\mathrm{s}, j}(v) h_{j, i}^{s \rightarrow s}(v)}{1+P_{\mathrm{p}, j}(v) h_{j, i}^{p \rightarrow s}(v)}\right)\right)
\end{aligned}
$$

Subject to:

$$
\begin{aligned}
& \text { - } \sum_{i=1}^{S_{j}} \sum_{v \in \mathcal{I}_{j} \cup \overline{\mathcal{I}}_{j}} \pi_{s, j}(v, i) P_{\mathrm{s}, j}(v) \leq \tilde{P}_{j}, \forall j=1, \cdots, N_{\mathrm{BS}} \\
& \text { - } \epsilon_{j}^{(\mathrm{p})} \pi_{s, j}(v, i) P_{\mathrm{s}, j}(v) h_{j, i}^{s \rightarrow p}(v) \leq I_{\mathrm{th}}, \forall i \in \overline{\mathcal{I}}_{j} \\
& \text { - } \mathcal{S}_{j}+\mathcal{P}_{j}^{\text {(roamed })} \leq \bar{C}_{s}, \forall j=1, \cdots, N_{\mathrm{BS}} \\
& \text { - } \Pi_{\mathrm{s}} \geq \Pi_{\mathrm{s}}^{\text {min }}, \\
& \text { - } \sum_{i=1}^{S_{j}} \pi_{s, j}(v, i) \leq 1, \quad \forall v \in \mathcal{I}_{j} \cup \overline{\mathcal{I}}_{j}, \forall j=1, \ldots, N_{\mathrm{BS}} .
\end{aligned}
$$

where $\mathcal{I}_{j}$ represents the set of free subcarriers belonging to the $j^{\text {th }}$ BS not allocated to the PN users while $\overline{\mathcal{I}}_{j}$ corresponds to the set of subcarriers used by the $j^{\text {th }}$ BS. $\boldsymbol{P}$ and $\boldsymbol{\pi}$ are the vectors containing the elements $P_{s, j}(v)$ and $\pi_{s, j}(v, i)$ representing the status of each subcarrier $v$ of the $j^{\text {th }}$ BS whether it is allocated to user $i$ (i.e., $\pi_{s, j}(v, i)=1$ ) or not (i.e., $\pi_{s, j}(v, i)=0$ ). The constraint (10) indicates that the SN's BS transmit power cannot exceed the BS power budget denoted by $\tilde{P}_{j}$. The parameter $I_{\text {th }}$ denotes the interference threshold tolerated by the PN during the spectrum sharing scenario as imposed in (11). The constraint (12) forces the number of served users by a BS to be less than the BS capacity. Finally, $\Pi_{\mathrm{s}}$ is the $\mathrm{SN}$ achieved profit that should be greater than the minimum required profits, denoted by $\Pi_{\mathrm{s}}^{\min }$ in (13). The constraint (14) indicates that a user can be served by only one subcarrier from one BS. Note that the total power budget of a BS used for secondary transmission is reduced during the spectrum leasing mode since priority is given to primary communication. Hence, the power budget is expressed as follows

$$
\tilde{P}_{j}=\bar{P}-\left(1-\epsilon_{j}^{(\mathrm{p})}\right) \sum_{i=1}^{\mathcal{P}_{j}^{\text {(roamed) }}} \frac{P_{\min }}{K} d_{j, i}^{\nu} h_{j, i}^{s \rightarrow p},
$$

where $\bar{P}$ is the fixed maximum BS power budget. On the other hand, the SN profit is expressed as follows

$$
\begin{aligned}
\Pi_{\mathrm{s}}= & \underbrace{\sum_{j=1}^{N_{\mathrm{BS}}} p_{\text {rate }} R_{s}}_{\text {Service revenue }}+\underbrace{\sum_{j=1}^{N_{\mathrm{BS}}}\left(1-\epsilon_{j}^{(\mathrm{p})}\right)\left(p_{\text {roam }} \mathcal{P}_{j}^{(\text {roamed })}-p_{\mathrm{sl}}\right)}_{\text {Collaboration profit }} \\
& -\underbrace{\sum_{j=1}^{N_{\mathrm{BS}}} \epsilon_{j}^{(\mathrm{p})} p_{\mathrm{ss}}-}_{\text {Non-collaboration cost }}-\underbrace{\sum_{j=1}^{N_{\mathrm{BS}}} p_{\mathrm{e}} P_{\mathrm{s}, j}}_{\text {Energy cost }},
\end{aligned}
$$

where $p_{\text {rate }}$ is the unitary cost of the secondary achieved rate. Note that the non-collaboration cost corresponds, here, to the spectrum sharing cost under the underlay mode. 


\section{PRIMARY BS ON/OFF SWITCHING AND THE SN RESOURCE AlLOCATION}

In this section, we describe the green collaboration mechanisms between the PN and the SN. As mentioned earlier, the optimal BS combination, as well as the SN power allocation at the different BSs, are determined at the common broker that proposes the determined collaboration schemes. The PN and the SN problems are combinatorial problems as their solutions cannot be determined analytically due to the existence of the binary variables $\epsilon^{(p)}$. Therefore, we propose to solve them into two steps. The first step is deciding which PN BSs should be switched off. The second step involves the determination of the power allocation of the SN.

\section{A. The Proposed PN Network Management}

We propose, in this part, a suboptimal algorithm involving switching off a certain number of BSs without falling below the PN or the SN minimal profits or the PN outage. The proposed algorithm starts by computing different PN and SN revenues for the two extreme cases, i.e., the case when all the PN BSs are switched on, denoted by "All On" and the case when all the PN BSs are switched off, denoted by "All Off". We compute for each $j^{\text {th }}$ BS the corresponding primary and secondary revenue when it is switched on or off. The next step is to switch off the BSs one by one and to check, in each step, whether or not the constraints are infringed. If so, the last switched off BS should be kept active. However, the BSs to be switched off should be chosen smartly in order to reach a solution close to the optimal one. Intuitively, the first BS to be switched off is the one that consumes the highest energy and produces the lowest profit then do the same for the remaining BSs if the constraint is respected. Note that, with this method, if switching off a BS infringes the minimum profit constraint, this BS and the remaining BSs should remain active since any other active BS will infringe the profit constraint. However, the BS with minimum profit does not necessarily correspond to the one with maximum energy. Consequently, we define the profit per energy metric as the ratio between the profit and the energy (\$/Watt). Then, we propose to switch off the BS with the minimum profit per energy, i.e. the BS that provides the least profit per Watt. Our proposed sub-optimal BS on/off switching is summarized in Algorithm 1

\section{B. Secondary Resource Allocation}

In order to optimize the power allocation of the $\mathrm{SN}$, we assume that the value of $\epsilon^{(p)}$ is given. Thus, the number of primary roamed users $P_{j}^{\text {(roamed) }}$ in addition to the power allocated per each active primary BS could be determined using (3). Hence, $P_{\mathrm{p}, j}(i), \forall i, j$ and $\tilde{P}_{j}$ are known and the SN's optimization problem could be solved using the dual decomposition method [11] as it is satisfying the dual time sharing condition investigated in [11]. Thus, the duality gap of the non-convex resource allocation problem in the multicarrier system is negligible as the number of subcarriers is sufficiently large compared to the number of users. Hence, the dual

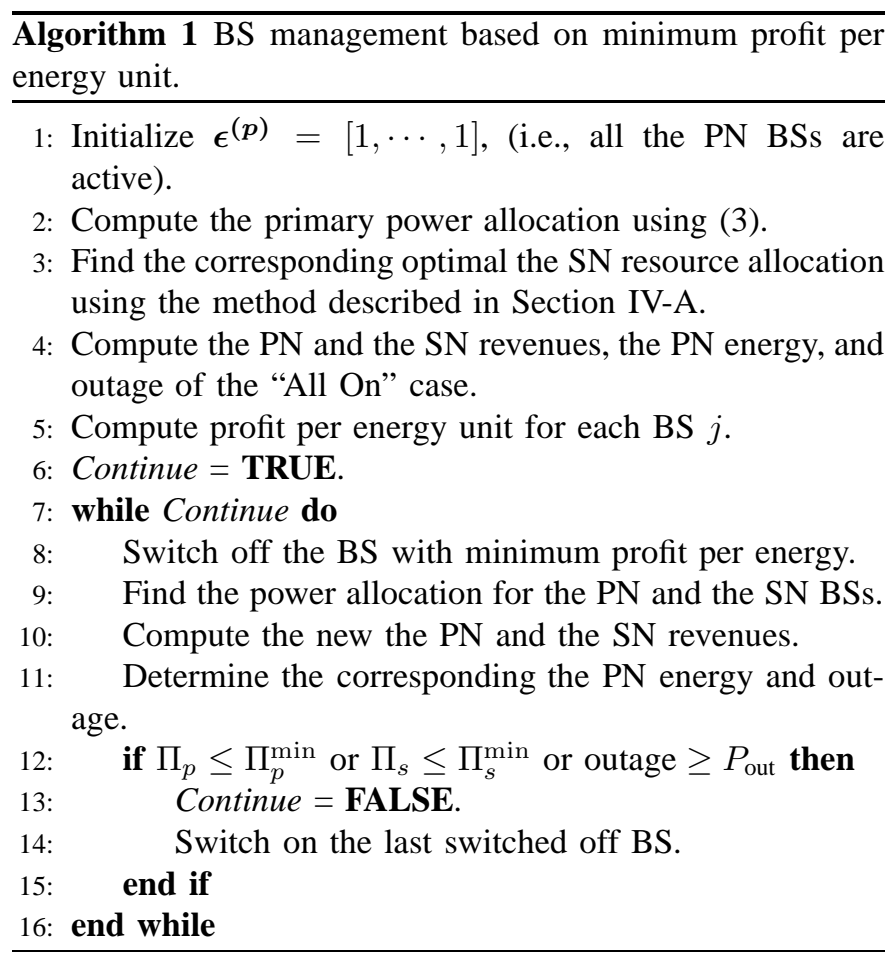

optimization problem associated with the primal problem is given by

$$
\begin{aligned}
& \underset{\boldsymbol{\lambda}, \mu \geq 0}{\operatorname{maximize}} g(\boldsymbol{\lambda}, \mu), \\
& \text { subject to: }
\end{aligned}
$$

where $\boldsymbol{\lambda}=\left[\lambda_{1}, \lambda_{2}, \ldots, \lambda_{N_{\mathrm{BS}}}\right]$ is the Lagrangian vector that contains the Lagrangian multipliers associated to constraints (10) while $\mu$ is the Lagrangian multiplier related to constraint (13). The dual function $g(\boldsymbol{\lambda}, \mu)$ is defined as follows

$$
g(\boldsymbol{\lambda}, \mu)=\underset{\boldsymbol{\pi}, \boldsymbol{P} \geq 0}{\operatorname{minimize}} \mathcal{L}(\boldsymbol{\lambda}, \boldsymbol{\mu}),
$$

where $\mathcal{L}(\boldsymbol{\lambda}, \mu)$ is the Lagrangian function of the secondary optimization problem. The steps to solve the dual problem can be described as follows:

- Step 1: Initialize the Lagrangian multipliers $\boldsymbol{\lambda}$ and $\mu$.

- Step 2: Find the optimal value of $P_{s, j}(v)$ for each pairs $(j, v)$ by solving the problem (17) for the fixed Lagrangian multipliers and $\epsilon_{j}^{(\mathrm{p})}=1$. Hence, the optimal power $P_{s, j}^{*}(v)$ can be given as follows

$P_{s, j}^{*}(v)=\left\{\begin{array}{c}\left.\min \left\{\left[\frac{1}{\lambda_{j}}-\frac{1+P_{\mathrm{p}, j}(v) h_{j, i}^{p \rightarrow s}(v)}{h_{j, i}^{s} \vec{h}^{s}(v)}\right)\right]^{+}, \frac{I_{\mathrm{th}}}{h_{j, i}^{s \rightarrow p}(v)}\right\}, \forall i \in \overline{\mathcal{I}}_{j}, \\ {\left[\frac{1}{\lambda_{j}}-\frac{1}{h_{j, i}^{s \rightarrow}(v)}\right]^{+}, \forall i \in \mathcal{I}_{j}}\end{array}\right.$ where $[x]^{+}=\max (0, x)$.

- Step 3: Substitute the optimal powers derived in (19) into (17). Thus, we obtain a linear assignment problem with respect to elements $\pi_{s, j}(v, i)$ and can be solved efficiently by using the Hungarian algorithm [12]. The solution obtained by the dual method is an asymptotically optimal solution [11].

- Step 4: After finding the optimal solutions $P_{s, j}(v)^{*}$ and $\pi_{s, j}(v, i)^{*}$ corresponding to the initialized Lagrangian multipliers in Step 1, we can employ the subgradient 
method to find their optimal values and thus the optimal solution of the problem [13]. Hence, to obtain the solution, we can start with any initial values for the Lagrangian multipliers and evaluate the optimal solutions (i.e., $P_{s, j}(v)^{*}$ and $\left.\pi_{s, j}(v, i)^{*}\right)$. We then update the Lagrangian multipliers at the next iteration $(i+1)$ as follows

$$
\lambda_{s}^{(i+1)}=\lambda_{s}^{(i)}-\delta^{i}\left(\tilde{P}_{j}-\sum_{i=1}^{S_{j}} \sum_{v \in \mathcal{I} \cup \overline{\mathcal{I}}_{j}} \pi_{s, j}(v, i) P_{\mathrm{s}, j}(v)\right), \forall j,
$$

$$
\mu^{(i+1)}=\mu^{(i)}+\varpi^{i}\left(\Pi_{\mathrm{s}}\left(P_{s, j}(v), \pi_{s, j}(v, i)\right)-\Pi_{\mathrm{s}}^{\min }\right),
$$

where $\delta^{i}$ and $\varpi^{i}$ are the updated step size according to the non-summable diminishing step length policy (see [13] for more details). The updated values of the optimal solution and the Lagrangian multipliers are repeated until convergence.

\section{Numerical Results}

We consider a geographical area covered by the PN and the SN possessing $12 \mathrm{BSs}$. The users distribution over the area could follow a uniform distribution. Table 1 summarizes the different parameters and prices adopted in our numerical results. We denote by $\$$ the monetary unit.

TABLE I: Adopted prices and network parameters.

\begin{tabular}{|l|c||l|c|}
\hline Parameter & Value & Parameter & Value \\
\hline \hline Number of BSs & 12 & Cell radius $(\mathrm{m})$ & 500 \\
\hline Number of subcarriers & 50 & Allowed outage $P_{\text {out }}$ & $5 \%$ \\
\hline Sharing price $p_{\text {ss }}(\$)$ & 30 & Leasing price $p_{\text {sl }}(\$)$ & 30 \\
\hline Service price $p_{\text {op }}(\$)$ & 2 & Rate price $p_{\text {rate }}(\$)$ & 0.75 \\
\hline Roaming price $p_{\text {roam }}(\$)$ & 2 & Energy price $p_{\mathrm{e}}(\$)$ & 0.15 \\
\hline Minimal profit percentage $\tau$ & $90 \%$ & BS transmit power $(W)$ & 2 \\
\hline Minimal power $P_{\min }(\mathrm{dB})$ & -10 & Scaling parameter $a$ & 7.84 \\
\hline BS constant power $b(W)$ & 71.5 & Interf. threshold $I_{\text {th }}(\mathrm{dB})$ & 0 \\
\hline Pathloss exponent $\nu$ & 3.76 & Pathloss constant $K$ & $10^{-4}$ \\
\hline
\end{tabular}

In Fig. 2, we plot the performances of the PN and the $\mathrm{SN}$ as a function of the roaming price. In Fig. 2, we study
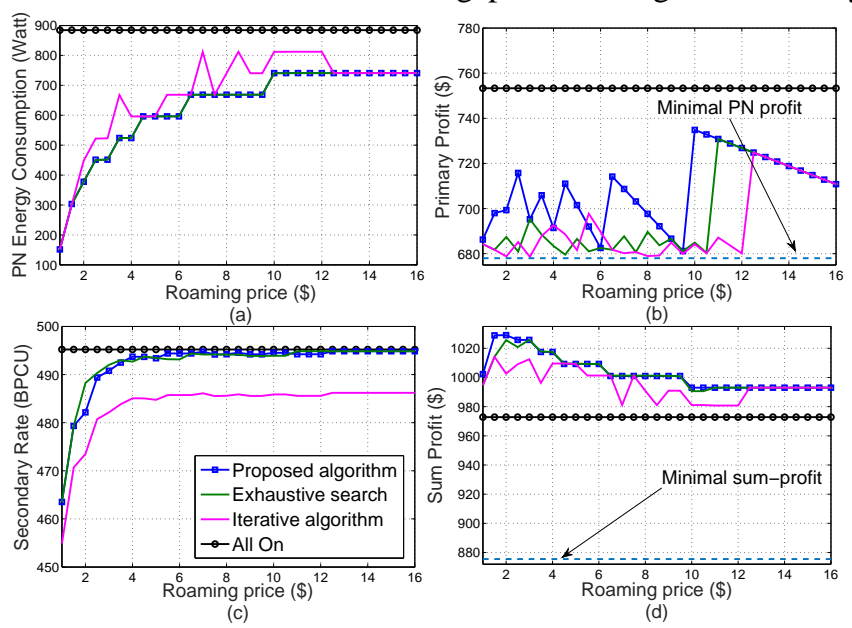

Fig. 2: Evolution of the PN and the SN performances with the roaming price variation.

the effect of the roaming price, $p_{\text {roam }}$ on the $\mathrm{PN}$ and the SN performances. In Fig. 2.a, we show that the PN energy consumption increases with $p_{\text {roam }}$ under a discrete stagnation levels. This slope of the energy consumption is related of the number of BSs switched on. In fact, as $p_{\text {roam }}$ increases, the profit decreases, and the PN needs to switch on an additional BS to meet the profit requirement. In Fig. 2, we also compare the performance of the proposed algorithm with those of the ES method and an iterative algorithm presented in [14] and denoted by 'IA'. ES consists in evaluating the achieved utility functions for all possible combinations and then selecting the combination providing the minimum PN energy consumption while respecting the $\mathrm{PN}$ and the $\mathrm{SN}$ profit constraints. This method is very complex mainly for large-scale networks since it requires $2^{N_{\mathrm{BS}}}$ tests till reaching the optimal solution. IA is a less complex approach consisting in turning off one BS every iteration. The selected BS is the one, when it is turned off, helps the PN achieving the lowest energy consumption without compromising the profit constraints of both networks. The algorithm converges when no more BSs can be eliminated as their elimination will affect the system constraints. Hence, IA needs $N_{\mathrm{BS}}^{2}$ tests before reaching its suboptimal solution (For more details, see [14]).

We show that our algorithm performances are very close to ES, whereas IA achieves higher energy consumption. This is because IA turns off at each iteration the BS having the highest transmit power consumption as at each time, it eliminates the BS that contributes the most to the network energy reduction. Hence, the BSs with the highest number of users are turned off first.

In Fig. 2.b, we plot the PN profit as a function of $p_{\text {roam, }}$ and we notice that the ES algorithm gives the closest profit to the minimal profit whereas our algorithm is relatively far. However, since the corresponding energy consumption of the two algorithms is almost the same, our algorithm is considered to be better than the ES in terms of profit and low-complexity.

In Fig. 2.c, we show that the $\mathrm{SN}$ rate is increasing when $p_{\text {roam }}$ increases which mean that the roaming price enhances the achievable $\mathrm{SN}$ rate. In addition, the maximum $\mathrm{SN}$ rate that can be achieved is the rate of the "All On" case since the rate used in serving the roamed PUs is not considered in the SN rate. In Fig. 2.d, we plot the SN profit and we show that it decreases with $p_{\text {roam }}$. This contradictory observation can be explained by the fact that when $p_{\text {roam }}$ is high, the PN needs to switch on more BSs to meet its profit requirement which means lower roaming revenue in addition to the spectrum sharing cost. This fact means that $p_{\text {roam }}$ is a key parameter that affects both the PN and the SN performances.

In Fig. 3, we show that the sum profit of both the PN and the $\mathrm{SN}$ is also decreasing with the energy price, $p_{\mathrm{e}}$. However, we show that the sum profit of our proposed algorithm is either equal or higher than the sum profit of the case of no cooperation, i.e. "All On". Consequently, cooperation between the PN and the SN produces a higher global profit even if the problem is not about maximizing the profit.

In Fig. 4, we analyze the energy price effect on the PN energy consumption and profit. In Fig. 4.a, we show that the PN profit and the minimal profit decrease linearly with the energy price where the minimal profit has the steepest slope. When the energy price increases to a certain level, the PN is 


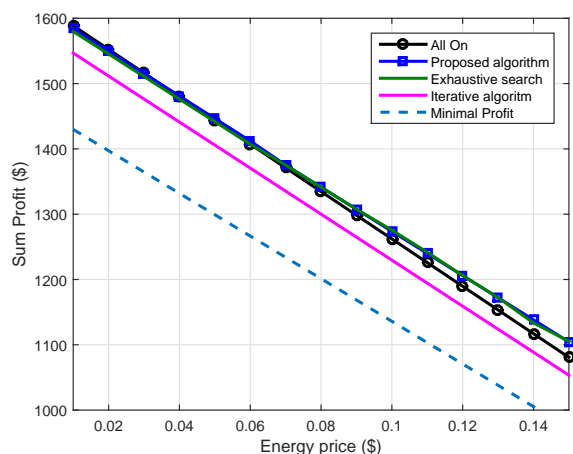

Fig. 3: Sum profit versus the energy price
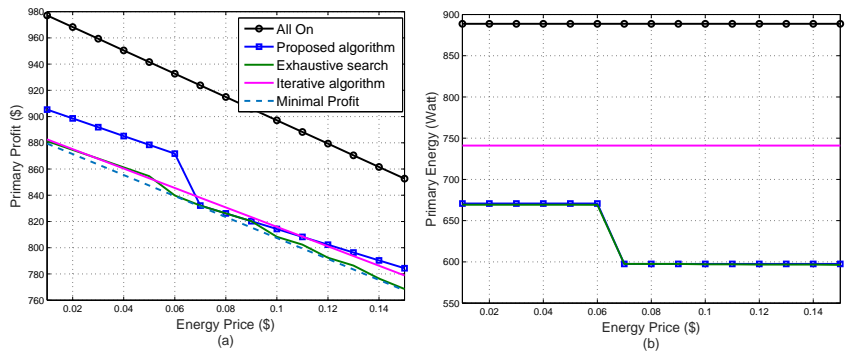

Fig. 4: Primary energy consumption and profit versus the energy price.

able to switch off an additional BS as the corresponding profit stays above $\Pi_{\mathrm{p}}^{\min }$ which also corresponds to a drop in energy consumption as shown in Fig. 4.b. In Fig. 4.b, we also show that as far as the same number of BSs are switched on, the energy consumption is not changing. After deactivation of the BS, the drop in energy consumption reaches a new constant level regardless of the variation of the energy price.

In Fig. 5, we plot the $\mathrm{SN}$ rate as a function of the PN energy consumption with various values of $p_{\text {roam }}$ to show the performance region. Overall, there is a trade-off between the $\mathrm{PN}$ energy and the SN rate. Note that the best region is the upper-left in which the PN energy is minimized, and the SN rate is maximized. Note that the non-cooperation "All On" case is located in the upper right part with high the PN energy

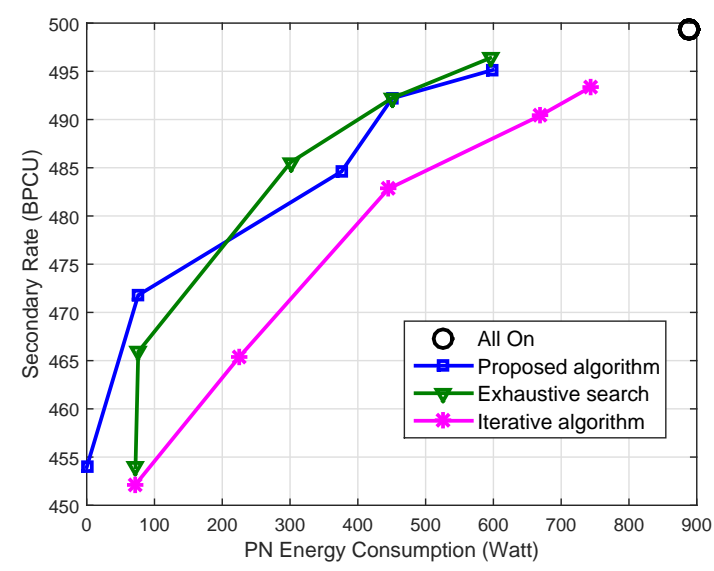

Fig. 5: PN energy versus the $\mathrm{SN}$ rate with various values of consumption and high the SN rate. We show that our proposed algorithm is close to the ES and offers better regions at low rate-energy.

\section{CONCLUSION}

In this paper, we addressed a novel energy-saving management for cellular networks in the cognitive radio spectrum trading framework. The novel cells management is based on switching off some of the primary network (PN) base stations (BSs) without affecting profit or outage constraints. The PN offloads its users to the secondary network (SN) that receives a roaming revenue in return. The SN either shares or leases the spectrum if available and maximizes its rate. We propose a low-complexity algorithm based on switching off the BSs with the minimal profit per energy unit if the profit and outage constraints are respected for both the PN and the SN. In the numerical results, we showed that our proposed on/off switching approach performs close to the high-complexity exhaustive search. We also show that the roaming price is an important parameter to decide either or not to switch off the PN BSs.

\section{REFERENCES}

[1] Y. Liu, L. Cai, X. Shen, and H. Luo, "Deploying cognitive cellular networks under dynamic resource management," IEEE Wireless Communications, vol. 20, no. 2, pp. 82-88, Apr. 2013.

[2] Nokia Networks, "5G use cases and requirements," White paper, 2014.

[3] Z. Hasan, H. Boostanimehr, and V. Bhargava, "Green cellular networks: A survey, some research issues and challenges," IEEE Communications Surveys Tutorials, vol. 13, no. 4, pp. 524-540, Fourth 2011.

[4] "Spectrum policy task force," Federal Communications Commission, Tech. Rep. ET Docket no. 02-135, Nov. 2002.

[5] J. Mitola and G. Q. Maguire, "Cognitive radio: Making software radios more personal," IEEE. Personal Communications, vol. 6, no. 4, pp. 1318, Aug. 1999.

[6] S. Haykin, "Cognitive radio: Brain-empowered wireless communications," IEEE Journal on Selected Areas in Communications, vol. 23, no. 2, pp. 201-220, Feb. 2005.

[7] R. Thomas, L. DaSilva, and A. MacKenzie, "Cognitive networks," in First IEEE International Symposium on New Frontiers in Dynamic Spectrum Access Networks (DySPAN'05), Maryland, USA., Nov. 2005, pp. 352-360.

[8] Y. Guo, J. Xu, L. Duan, and R. Zhang, "Joint energy and spectrum cooperation for cellular communication systems," IEEE Transactions on Communications, vol. 62, no. 10, pp. 3678-3691, Oct. 2014.

[9] H. Ghazzai, E. Yaacoub, M.-S. Alouini, and A. Abu-Dayya, "Optimized smart grid energy procurement for LTE networks using evolutionary algorithms," IEEE Transactions on Vehicular Technology, vol. 63, no. 9, pp. 4508-4519, Nov. 2014.

[10] A. Fehske, P. Marsch, and G. Fettweis, "Bit per joule efficiency of cooperating base stations in cellular networks," in IEEE GLOBECOM Workshops (GC Wkshps 2010), Dec. 2010, pp. 1406-1411.

[11] W. Yu and R. Lui, "Dual methods for nonconvex spectrum optimization of multicarrier systems," IEEE Transactions on Communications, vol. 54, no. 7, pp. 1310-1322, Jul. 2006

[12] H. W. Kuhn, "The Hungarian Method for the Assignment Problem," 50 Years of Integer Programming 1958-2008. Berlin, Heidelberg, ch2. 2010.

[13] S. Boyd and A. Mutapcic, "Stochastic Subgradient Methods," Notes for EE364, Stanford University, Winter. 2006-07.

[14] H. Ghazzai, E. Yaacoub, M. Alouini, and A. Abu-Dayya, "Optimized green operation of LTE networks in the presence of multiple electricity providers," in IEEE Globecom Workshops, Anaheim, CA, USA, Dec. 2012, pp. 664-669. 